\title{
Practical Experience of Insulation Condition Evaluation in an On-site HV Installation Appliying a PD Measuring Procedure
}

\author{
F. Álvarez , F. Garnacho , R. Albarracín , R. Granizo , M. Dong and J. Ortego
}

\begin{abstract}
Analysis of the types of defects and the degradation modes in different insulation materials of highvoltage (HV) electrical systems has shown that the presence of partial discharges (PD) is a very common characteristic in all of them. Once PD activity is detected, the identification and localization of the associated type of defect is very important to evaluate whether the discharges are harmful or not. During the last two decades, different approaches have been developed and applied to PD measurement and processing techniques to deal with the most common problems arising in on-site and on-line measurements, such as: high levels of background noise, appearance of interferences, simultaneous presence of different PD sources and the difficulty of locating the site of the defects and to identify them. This paper proposes a measuring method together with the implementation of three signal processing tools in order to overcome all these difficulties that appear in on-site and on-line tests. The measuring method has been implemented to evaluate in an on-site practical experience the insulation condition of the power equipment in a GIS substation. After the application of the method, important insulation defects were detected, identified and localized, on time to avoid uncontrolled failures in the electrical system of the substation under test.
\end{abstract}

\section{INTRODUCTION}

PD measurements provide valuable information for assessing the condition of the HV insulation systems of power cables, power transformers and GIS substations [1-3], contributing to their quality assurance. Experiences about commissioning and maintenance tests performed by means of mobile generators and on-line in HV installations are shown in many previous studies and some of them are summarized in [4] Many different on-line PD techniques have been developed in order to assess the insulation condition of operating equipment [5-8]. For utilities, the most attractive characteristics of on-line measurements are that once the sensors are installed in the grid, the electrical service is not interrupted and that electrical systems are tested in real operating conditions. This paper is focused on the presentation of a real case study of on-line PD measurements applying a particular measuring method. In next section, the method and processing tools used are presented and in section III, the practical case is discussed and analysed in detail.

\section{MeAsuring PRocedure AND PROCESSING TOOLS}

Different PD measuring techniques specially designed to perform on-line measurements in GIS substations have been developed in the last years. Non-conventional electromagnetic PD methods operating in ultra-high-frequency (UHF) bands are usually used when this type of tests are carried out $[9,10]$. In PD measurements the signal acquisition, the subsequent signal-processing and the capability to obtain an accurate diagnosis are conditioned by the selection of a suitable detection technique and by the implementation of effective signal-processing tools. The measuring and processing method proposed in this paper is based on the combined implementation of wideband PD measurements performed in the high-frequency (HF) and UHF frequency ranges together with the use of three processing tools. The method involves several steps which are presented in the following flow-chart.

1. Realization of synchronized PD measurements in the HF and UHF frequency ranges.

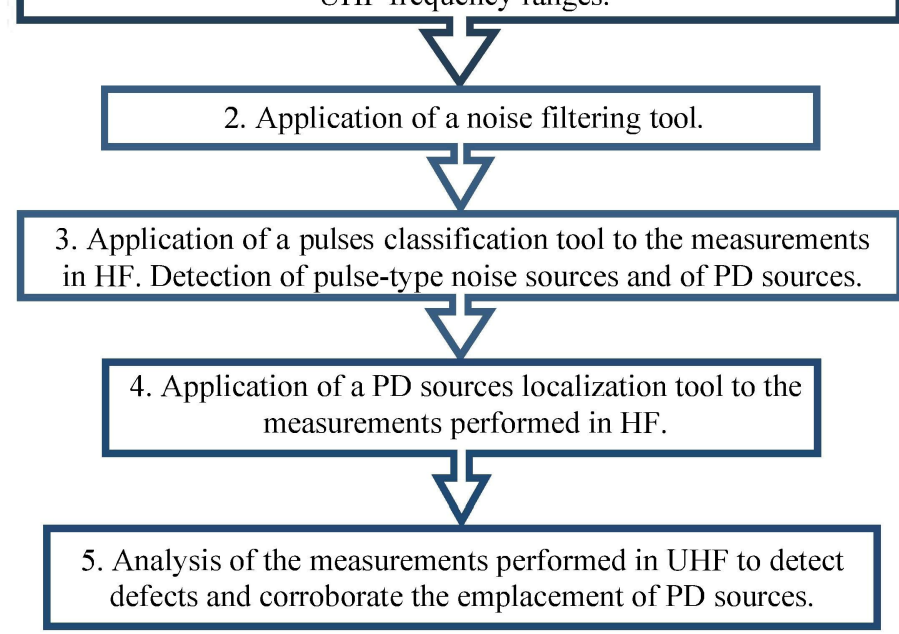

Fig. 1. Flow-chart of the measuring and processing method proposed

The noise filtering tool used in the $2^{\text {nd }}$ step is based on the wavelet transform (WT) and enables the separation of pulse-shaped signals generated by PD or noise sources from background noise [11]. The classification tool applied in the $3^{\text {rd }}$ step draws on the waveform analysis of the registered pulses. This processing tool is useful to discriminate different PD sources or pulse-type noise sources present in the same $\mathrm{HV}$ installation. The classification technique is based on the characterization of each pulse with three parameters: the 
predominant oscillation frequency $f$ and two waveform parameters, $\alpha$ and $\beta$, that define the asymmetry of the enveloping function $h(t)$ (1) that fits the local maxima of the absolute values of the recorded signals.

$$
h(t)=\frac{A}{e^{\alpha(t)}+e^{-\beta(t)}}
$$

The $3 \mathrm{D}$ representation of these three parameters allows the identification of different PD clusters [11]. Finally, the automatic location tool applied in step $4^{\text {th }}$ is based on the analysis of the time delay $\left(\Delta t_{i}=t_{i 2}-t_{i 1}\right)$ between the times of arrival of the pulses $\left(t_{i 2}\right.$ and $\left.t_{i 1}\right)$ to the HFCT sensors placed at a known distance $L$ in the HV installation. A pulse registered in one measuring unit is correlated with another registered in other only if the time delay between the arrival times of both pulses $\Delta t_{i}$ is less than the propagation time between sensors $t_{w}(2)$. On the basis of the knowledge of the propagation time $t_{w}$ (being $V$ the propagation speed of the signals) and the distance between sensors $L$, the location of a pulse source $x_{i}\left(\Delta t_{i}\right)$ with respect to the position of a measuring unit is established by expression (3) [11].

$$
\begin{gathered}
t_{w}=L / V \\
x_{i}\left(\Delta t_{i}\right)=\frac{L}{2} \cdot\left[1-\left(\frac{2 \cdot \Delta t_{i}}{t_{w}}\right)\right]
\end{gathered}
$$

\section{PRACTICAL EXPERIENCE}

In this section a case study of PD measurements performed in a real HV electrical system is presented. With the aim of diagnosing the insulation condition of the elements composing the proposed installation, the measurement procedure presented in the previous section is applied. The electrical system analysed comprises the following elements of a GIS substation of the primary distribution net located within an urban area:

- Two power transformers of 45/15 kV and 25 MVA of rated power each, insulated with mineral oil.

- A set of $45 \mathrm{kV}$ distribution switchgears insulated with $\mathrm{SF}_{6}$.

- And the respective interconnecting cables between the transformers and the switchgears, insulated with XLPE.

Fig. 2 shows the layout of the installation where the PD tests were performed.

The measurements were carried out on-line with the HV installation operating under normal conditions. Due to the operating conditions of the GIS substation, in this occasion the electrical utility company only allowed to install PD sensors on the transformers side for the accomplishment of the measurements. Thus, it was not possible to install any PD sensor in the $45 \mathrm{kV}$ switchgears or in the cable terminals connected to them.

The tests were made with a PD measuring instrument specially designed to perform on-site and on-line PD measurements. This instrument is equipped with an acquisition board of 14 bits of vertical resolution, a bandwidth of $50 \mathrm{MHz}$ and a sampling frequency of $100 \mathrm{MS} / \mathrm{s}$. The signal processing tools described in the previous section are integrated in the processing and analysis stages of this measuring device in order to improve the ability to perform accurate diagnosis. The equipment is controlled by a computer connected via fiber optic cable or through wireless connection.

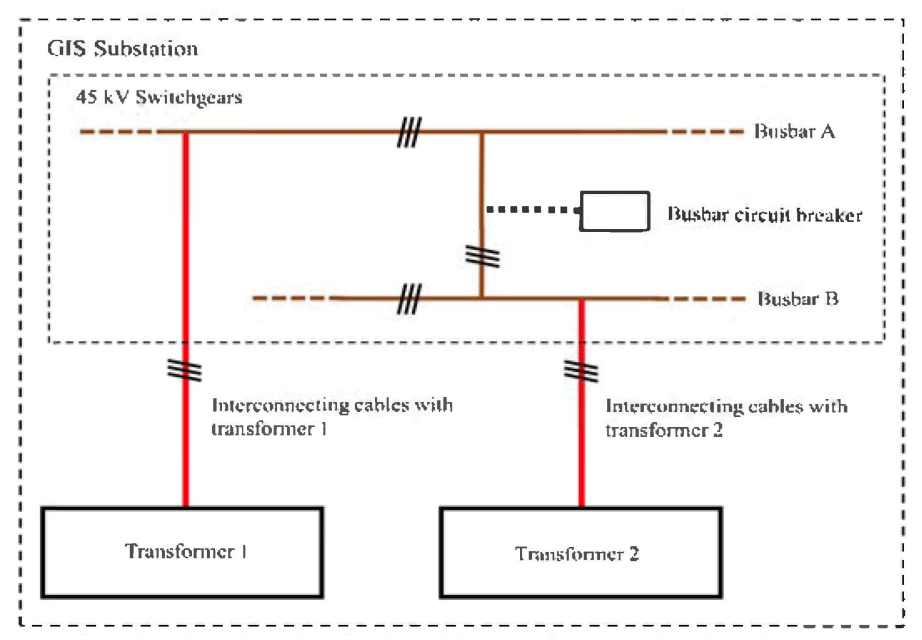

Fig. 2. Overall view of the electrical HV installation analysed with both power transformers, distribution switchgears and the interconnecting cables inside the GIS substation

The PD measuring instrument, designed to measure in the HF range, can be used to record and process signals measured in the UHF range, once they are transformed into HF signals by means of a frequency coniverter. The UHF-HF converter used in this case study enables to take the advantages of capturing the pulses in UHF and in turn record and analyse the signals with the same measuring instrument designed to perform PD measurements in HF. This converter is integrated with an internal amplifier whose gain can be adjusted and its bandwidth is from 0.1 to $3 \mathrm{GHz}$.

According with the measuring procedure (see Fig. 1) PD measurements must be performed in the HF and UHF frequency ranges. The PD measurements in the HF range were made with clamp-type high-frequency current transformer (HFCT) sensors and in the UHF range with non-invasive sensors, as there was not any invasive UHF sensor already installed in the HV components of the substation. The HFCT sensors and the UHF antennas were installed in all the cable terminals connected to the transformers, as shown in Fig. 3. Furthermore two HFCT sensors were also installed in the earth connection of the transformer tanks.
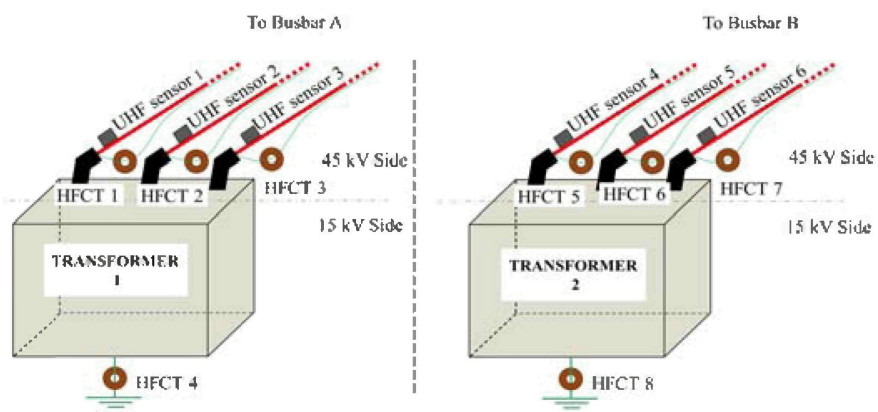

Fig. 3. Installation of the HFCT sensors in the earth connection of the cable terminals and power transformers and of the UHF antennas on the sheath of the cable terminals 
Fig. 4 shows in detail the installation of the sensors in one of the cable terminals and in Table 1 an overview of all the sensors used in the measurements and their position in the HV installation is presented.

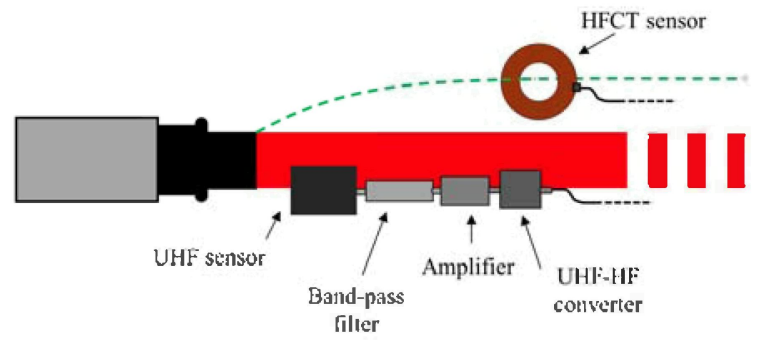

Fig. 4. Detail of the installation of the sensors in one of the cable terminals

TABLE I. LOCATION OF THE PD SENSORS USED IN THE INSTALLATION

\begin{tabular}{|c|c|c|c|}
\hline \multicolumn{2}{|c|}{$\begin{array}{c}\text { Interconnecting cables with } \\
\text { transformer } 1\end{array}$} & \multicolumn{2}{|c|}{$\begin{array}{c}\text { Interconnecting cables with } \\
\text { transformer } 2\end{array}$} \\
\hline HFCT sensors & Position & HFCT sensors & Position \\
\hline HFCT 1 & phase $\mathrm{R}$ & HFCT 5 & phase R \\
\hline HFCT 2 & phase $\mathrm{S}$ & HFCT 6 & phase $\mathrm{S}$ \\
\hline HFCT 3 & phase T & HFCT 7 & phase $T$ \\
\hline HFCT 4 & $\begin{array}{c}\text { earth } \\
\text { connection of } \\
\text { transformer } 1\end{array}$ & HFCT 8 & $\begin{array}{c}\text { earth } \\
\text { connection of } \\
\text { transformer } 2\end{array}$ \\
\hline UHF sensors & Position & UHF sensors & Position \\
\hline UHF 1 & phase $\mathrm{R}$ & UHF 4 & phase $\mathrm{R}$ \\
\hline UHF 2 & phase $S$ & UHF 5 & phase $\mathrm{S}$ \\
\hline UHF 3 & phase $\mathrm{T}$ & UHF 6 & phase $T$ \\
\hline
\end{tabular}

The HFCT sensors used (ref. DIAEL SEN-DO2) have a bandwidth of $100 \mathrm{kHz}$ to $20 \mathrm{MHz}$. The UHF sensors are noninvasive patch-type antennas (ref. IPEC OSM CCT2), with a bandwidth of 20 to $800 \mathrm{MHz}$. For PD measurements in UHF, where the noise level is very low, the use of an amplifier connected to the output of the sensors is recommended, since when low amplitude pulses are captured, this element helps to achieve more sensitivity when they are recorded by the measuring instrument. Furthermore, when the pulses present an amplitude slightly above the noise level, the use of an amplifier helps to differentiate in more detail a possible phase resolved PD (PRPD) pattern. In this study, the PD measurements with the UHF sensors have been performed with an amplifier of $20 \mathrm{~dB}$. This amplifier has a bandwidth from $100 \mathrm{kHz}$ to $1 \mathrm{GHz}$.

In addition, the measurements with the UHF antennas have been performed delimiting their bandwidth in a defined frequency range starting from $300 \mathrm{MHz}$. By doing this, a greater immunity to noise and interference signals of low and high frequency is obtained, being the signal-to-noise ratio (SNR) higher. Moreover, PD measurements in cable accessories from $300 \mathrm{MHz}$ allow a greater selectivity in the location of defects [11]. Considering the importance of these two advantages, a high-pass filter of $300 \mathrm{MHz}$ was connected at the output of the non-invasive UHF sensor. Besides, in order to avoid radiofrequency disturbances mainly generated by telecommunication applications [12], it is recommendable to implement also a low pass filter of $800 \mathrm{MHz}$. Fig. 4 shows the final configuration of a non-invasive sensor coupled on the sheath of one of the cable terminals with the band-pass filter (300-800 MHz), the amplifier and the frequency converter.

It is important to highlight that if an insulation defect were detected with the HFCT sensors, the measurements performed with the UHF sensors may be useful to determine or to corroborate the emplacement of the PD source.

After the installation of the sensors, synchronized PD measurements were performed (step 1 of the procedure). The measurements were made with four measuring instruments of four channels each one, and the synchronization between them was accomplished by the time reference of the coordinated universal time (CUT) from the pulse per second signal (PPS) of a global positioning system receiver (GPS).

Once the background noise filtering tool is applied to the measurements made with all the sensors (step 2 of the procedure), the next step consiists in removing the impulsional noise signals captured with the HFCT sensors and identify the number of PD sources involved in each measurement by applying the pulses classification tool (step 3).

After a preliminary analysis of all the PRPD patterns obtained, PD activity was detected in the measurements performed with the following sensors.

- In the interconnecting cables with transformer 1:

- HFCT 2 positioned in the earth connection of phase S

- HFCT 3 positioned in the earth connection of phase $\mathrm{T}$

- In the interconnecting cables with transformer 2:

- HFCT 7 positioned in the earth connection of phase $\mathrm{T}$

With the rest of HFCT sensors, only pulse-type noise signals of very low amplitude were detected and it was not visualized any characteristic PRPD pattern. Fig. 5 shows the positions of the $\mathrm{HV}$ installation where $\mathrm{PD}$ activity was detected. The PRPD patterns measured with the HFCT sensors in these positions are shown in Fig. 6. The result of the pulses classification tool (step 3) is shown in Fig. 7. In this case, the selection of the formed clusters enables to separate the PD pulses from the pulse-type noise signals. Fig. 8 shows the PRPD patterns corresponding to the clusters surrounded by the circles in Fig 7; the other clusters correspond to the pulse-type noise signals.

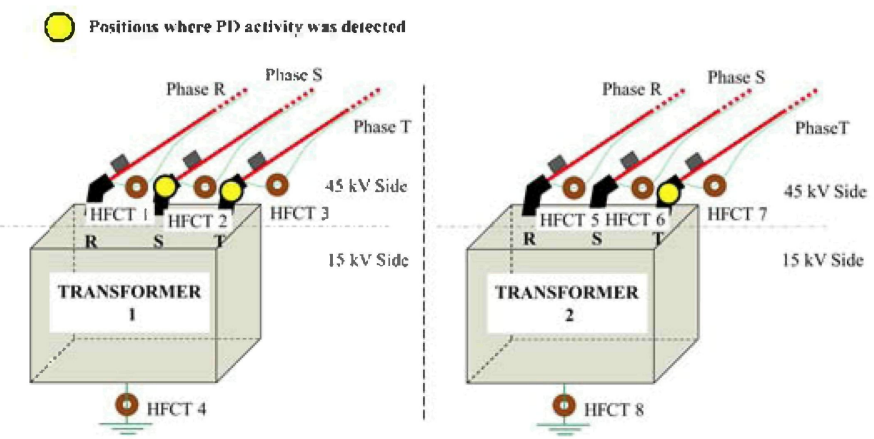

Fig. 5. Positions in the installation where PD activity was detected

Once applied the classification tool, in a first evaluation of these PRPD patterns it can be stated that only one PD source is present in each of them. Furthermore, all the patterns can be associated with internal defects as PD occur slightly before the zero crossings and in the increasing intervals of the test 
voltage, and also, there is a certain symmetry when comparing the patterns of both half-periods.

\begin{tabular}{|c|c|c|}
\hline \multicolumn{2}{|c|}{$\begin{array}{c}\text { INTERCONNECTING CABLES WITH } \\
\text { TRANSFORMER 1 }\end{array}$} & $\begin{array}{c}\text { INTERCONNECTING } \\
\text { CABLES WITH } \\
\text { TRANSFORMER 2 }\end{array}$ \\
\hline HFCT 2 in phase S & HFCT 3 in phase T & HFCT 7 in phase T \\
\hline & & \\
& &
\end{tabular}

Fig. 6. PRPD patterns obtained with the HFCT sensors.

\begin{tabular}{|c|c|c|}
\hline \multicolumn{2}{|c|}{$\begin{array}{c}\text { INTERCONNECTING CABLES WITH } \\
\text { TRANSFORMER 1 }\end{array}$} & $\begin{array}{c}\text { INTERCONNECTING } \\
\text { CABLES WITH } \\
\text { TRANSFORMER 2 }\end{array}$ \\
\hline HFCT 2 in phase S & HFCT 3 in phase T & HFCT 7 in phase T \\
\hline &
\end{tabular}

Fig. 7. 3D diagrams obtained with the pulses classification tool.

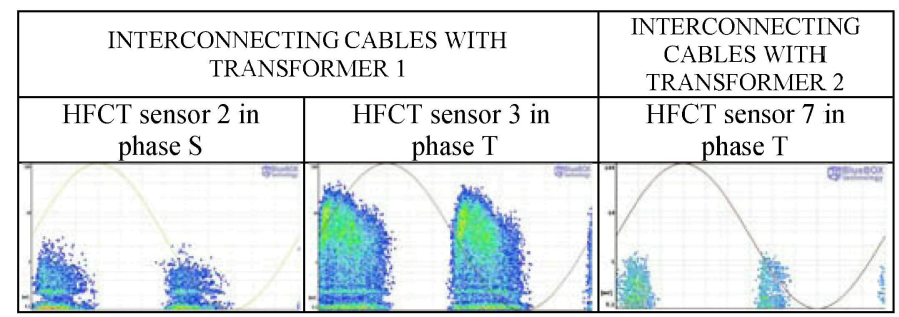

Fig. 8. PRPD patterns obtained after the elimination of the pulse-type noise signals.

The next step consists in the application of the automatic PD source location tool, with the aim to determine the position of the insulation defects (step 4). In this case, the PD location tool could only be applied to the synchronized measurements performed in phase $\mathrm{T}$ of the interconnecting cables because, as it is shown in Fig. 9, it was only in this phase where the PD pulses were detected in both cable ends.

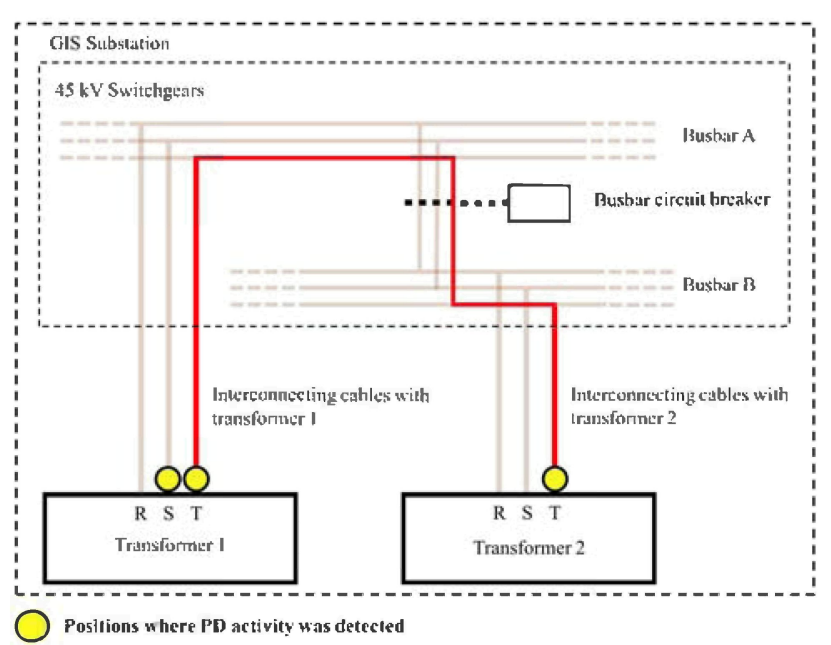

Fig. 9. PD detection with the HFCT sensors in phases $\mathrm{S}$ and T. The DP location tool can only be applied for the measurements performed in phase $\mathrm{T}$

The result of the localization tool applied to the PD detected in phase $\mathrm{T}$, after the analysis of the arrival times of the pulses to the HFCT sensors 3 and 7, is shown in Fig. 10.

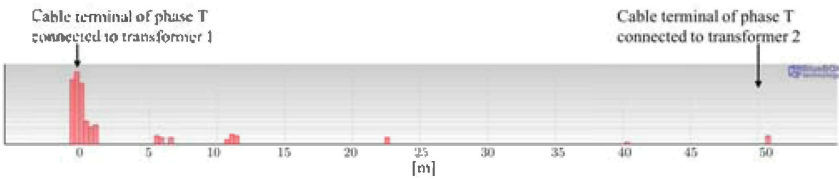

Fig. 10. PD mapping obtained with the location tool (distance between sensors $50 \mathrm{~m})$

In the PD mapping, only one emplacement corresponding to a PD source can be visualized. In this case, the PD source is positioned in the cable terminal connected to transformer 1 (in phase T).

In order to confirm that the PD source in phase $\mathrm{T}$ was in the terminal and not inside the transformer, and to know the localization of the defect in phase $S$, the measurements performed with the UHF sensors were analysed. PD activity was detected with sensors:

- In the interconnecting cables with transformer 1:

- UHF 2 positioned in the earth connection of phase $S$

$\circ \quad$ UHF 3 positioned in the earth connection of phase $T$

With the rest UHF sensors it was not detected any PRPD pattern. Fig. 11 shows the measured PD patterns in these positions.

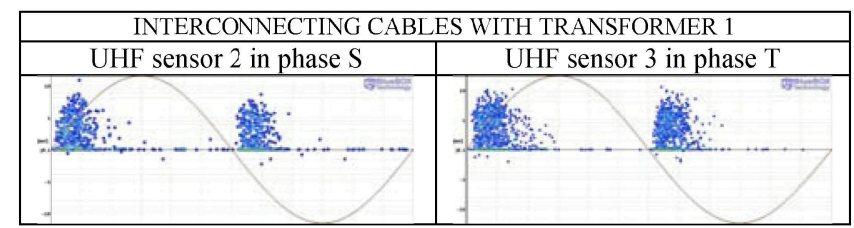

Fig. 11. PRPD patterns obtained with the UHF sensors in the terminals of phases $\mathrm{S}$ and $\mathrm{T}$ connected to transformer 1

Due to the selective approach of PD measurements in UHF when these are performed in cable accessories [11], it can be stated that the measured DP were generated in internal insulation defects positioned inside the cable terminals where the UHF sensors 2 and 3 were installed. This result allows confirming that the PD detected with the HFCT sensors located in these same terminals (of phases $\mathrm{S}$ and $\mathrm{T}$ ) are generated in the internal defects positioned inside them.

After the diagnosis, the utility decided to replace the cable terminals and in order to ratify the insulation defects, both accessories were disassembled. A significant degradation in the insulation parts of both terminals was detected. As an example, Fig. 12 shows the damaged areas of the terminal corresponding to phase $\mathrm{T}$, that evidenced an incorrect assembling of the accessory components. a)

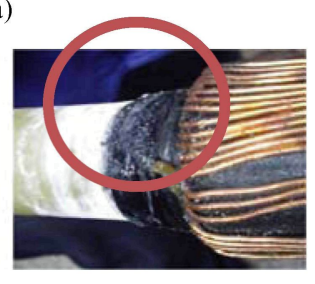

b)

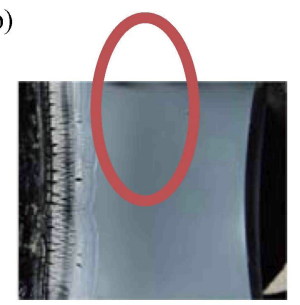

Fig. 12. a) Degradation on the surface of the cable main insulation b) Degradation in the enveloping silicone piece for the electric field control 


\section{CONCLUSIONS}

The proposed PD measuring and processing method applied in on-line PD measurements allows performing an accurate and reliable diagnosis of the insulation condition of HV installations.

The simultaneous and complementary measurements of $\mathrm{PD}$ in the HF and UHF ranges and the use in the proposed sequence of the three processing tools presented, enabled the identification and localization of the insulation defects present in a real HV installation.

For the measurements performed with UHF sensors the use of a UHF-HF converter enables to take the advantages of capturing the $\mathrm{PD}$ pulses in the UHF range and to process them in the HF range. Therefore, using only a PD instrument with a bandwidth covering the HF range, the signals measured with HF and UHF sensors can be analysed.

\section{ACKNOWLEDGMENT}

The authors would like to thank DIAEL Corporation for their continuous support in the development of the presented measuring and processing method integrated in the BlueBOX technology. 